\title{
Age Classification based on Corner Pixel Grey Level Co-Occurrences Matrix (CP-GLCM) of TN-LBP
}

\author{
Pullela S V V S R Kumar \\ Research Scholar, Acharya \\ Nagarjuna University, \\ Associate Professor, V.S. \\ Lakshmi Engineering College \\ for Women, Matlapalem, \\ Kakinada- 533461, India
}

\author{
J V R Murthy, Ph.D \\ Professor of CSE, University \\ College of Engineering, \\ Jawaharlal Nehru \\ Technological University \\ Kakinada, Kakinada- 533003, \\ India
}

\author{
V V Sahiti Srinidhi \\ B.Tech. III Year I Semester \\ CSE, SRM University, Chennai
}

\begin{abstract}
The present paper proposes a novel scheme based on third order neighbourhood LBP (TN-LBP). The present paper observed and noted that the TN-LBP forms two types of corner pixels i.e. top corner and bottom corner pixels. The present paper derived Grey Level Co-occurrence Matrix (GLCM) based on LBP values of Top Corner Pixels (TCP) of TN-LBP and Bottom Corner Pixels (BCP) of TN-LBP. On this GLCM features are derived. Based on these features human age is classified in to child ( 0 to 12 years) young adult (13 to 30 years), middle age ( 31 to 50 years) and senior age (above 60 years).
\end{abstract}

\section{General Terms}

Classification, Image Processing et. al.

\section{Keywords}

Age Classification, Local Binary Pattern (LBP). Third Order Neighborhood, Corner Pixel Grey Level Co-occurrence Matrix (CP-GLCM)

\section{INTRODUCTION}

A human face comprises of lot of information, and they can used in various applications like face recognition [3], age group classification [2]. Lot of research is undergoing in the area of human facial image processing and it is still active and interesting. The other research areas include predicting feature faces [4], reconstructing faces from some prescribed features [5], classifying gender, races, and expressions from facial images [6], and so on. On age group classification/estimation much less work has been done, but several applications such as enforcement of law in usage of certain types of drug and entertainment scenarios, targeted advertisements etc require require age group classification/estimation. Several applications are under development in the area of human communication to achieve automatic identification of individuals using computers.

Wen-Bing Horng, Cheng-Ping Lee and Chun-Wen Chen et.al [2] considered four age groups for classification, including babies, young adults, middle-aged adults, and old adults. This is implemented based on the symmetry of human faces and the variation of gray levels, the positions of eyes, noses, and mouths are located by applying the Sobel edge operator and region labeling in the above methods. Kwon et. al. [7] implemented age classification on facial images is based on cranio-facial development theory and skin wrinkle analysis in which only three age-groups babies, young adults, and senior adults.
Various age group classification method are implemented to classify facial images into various age groups i.e. babies and adults [8], two age groups 20-39 and 40-49 [9]. In addition to this classification of facial images based on sex $[9,10]$ are also implemented. Sasikiran et.al. [11] implemented age classification by reducing the image dimensionality and classified the human age into five categories. The above method is extended based on the topological texture features of the facial skin for an effective age classification that classified the human age into five groups [12]. Various age classification methods based on LBP are also proposed [14, 15] and a pattern based dimensionality reduction model for age classification is also proposed recently and classified age groups effectively into four groups [13].. The present paper attempted to classify the age groups into four categories based on Corner Pixel Grey Level Co-occurrence Matrix (CPGLCM) features extracted on TN-LBP on the facial image.

\section{DERIVATION OF CORNER PIXEL GREY LEVEL CO-OCCURRENCES MATRIX (CP-GLCM) OF TN-LBP}

The proposed method evaluated GLCM features on TCP (Top Corner pixels) and BCP (Bottom Corner Pixels) of TN-LBP. The proposed method based T-TN-LBP consists of 9 steps as described below.

Step 1: Take facial image as Input Image (Img).

Step 2: Convert the RGB image into Grey scale Image by using HSV color model.

Step 3: Crop the grey scale image.

Step 4: The present research evaluated TN-LBP on each 5 x 5 sub image. The TN contains only 13 pixels of 25 pixels of $5 \times 5$ neighborhoods as shown in Fig.1. The TN-LBP grey level sub image is converted into binary sub image by comparing the each pixel of TN grey level sub image with the mean value of $\mathrm{TN}$ grey sub image. The following Eqn.1 is used for grey level to binary conversion.

$$
\text { TN-P } P_{i}=\left\{\begin{array}{lll}
0 & \text { if } & P_{i}<V_{0} \\
1 & \text { if } & P_{i} \geq V_{0}
\end{array}\right\} \text { for } i=1,2, . .13
$$

Where $\mathrm{V}_{0}$ is the mean of the TN matrix. 


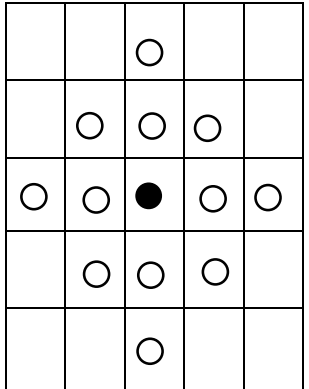

Fig. 1: Third Order Neighborhood for a central pixel.

Step 5: The interesting thing in TN-LBP is it will have two corner pixel patterns. The present research named them as TCP and BCP. Both TCP and BCP will have four pixels only. TCP of TN-LBP is indicated by green color and BCP of TNLBP are indicated by sky blue color in the Fig.2. The Pixels P1, P5, P13 and P9 form TCP of TN-LBP and the pixels P3, P6, P8 and P11 forms BCP of TN-LBP.

\begin{tabular}{|l|l|l|l|l|}
\hline & & P1 & & \\
\hline & P2 & P3 & P4 & \\
\hline P5 & P6 & P7 & P8 & P9 \\
\hline & P10 & P11 & P12 & \\
\hline & & P13 & & \\
\hline
\end{tabular}

Fig. 2: Considered diamond patterns

Step 6: LBP code is evaluated on the TCP and BCP of TNLBP. To achieve rotational invariance the minimum code is taken. The LBP code ranges from 0 to 15 .

Step 7: The Corner Pixel Grey Level Co-occurrence Matrix (CP-GLCM) of TN-LBP is generated by representing the TCP-pattern values on $\mathrm{X}$ - axis and $\mathrm{BCP}$-pattern values on $\mathrm{Y}$ axis. This method has the elements of relative frequencies in both patterns, since the LBP code values of these patterns i.e. TCP and $\mathrm{BCP}$ ranges from 0 to 15 . That is the reason the $\mathrm{CP}-$ GLCM of TN-LBP have a fixed size of $16 \times 16$ because of number of distinct values in this method is 16 .

Step 8: Extract the contrast, correlation, homogeneity and energy features on CP-GLCM of TN-LBP.

$$
\begin{aligned}
& \text { Energy }=\sum_{i, j=0}^{N-1}-\ln \left(P_{i j}\right)^{2} \\
& \text { Contrast }=\sum_{i, j=0}^{N-1} P_{i j}(i-j)^{2} \\
& \text { Homogenity }=\sum_{i, j=0}^{N-1} \frac{P_{i j}}{1+(i-j)^{2}} \\
& \text { Correlation }=\sum_{i, j=0}^{N-1} P_{i j} \frac{(i-\mu)(j-\mu)}{\sigma^{2}}
\end{aligned}
$$

Step 9: Based on four feature values facial image is classified as one of the category (Child Age(0-12), Young Age(13-30), Middle Age(31-50) and Senior Age (51 -70).

\section{RESULTS AND DISCUSSIONS}

In implementing this a database is established by collecting 1002 facial images from FG-NET database, 500 images from Google database and other 600 images from the scanned photograph which results in a total of 2102 sample facial images. In the proposed method the sample images are grouped into four age groups of Child age(0-12), Young Age(13-30), Middle Age(31-50) and Senior Age (51 -70). A few of them are shown in Fig.3. The statistical features are extracted from CP-GLCM of TN-LBP for different facial images and the results are stored in the feature database. Feature set leads to representation of the training set. The statistical features of four age groups of facial images are shown in tables $1,2,3$, and 4 respectively. Based on the derived features on CP-GLCM of TN-LBP an algorithm is derived by the present research to classify the facial image into one of the category of Child age $(0-12)$, Young Age(1330), Middle Age(31-50) and Senior Age (51 -70).

To evaluate the efficacy of the proposed method various facial images are considered and CPCM of TN-LBP is evaluated and derived the GLCM features on them. Based on the proposed age classification algorithm the age classification rate of the test images is established and noted in table 5.
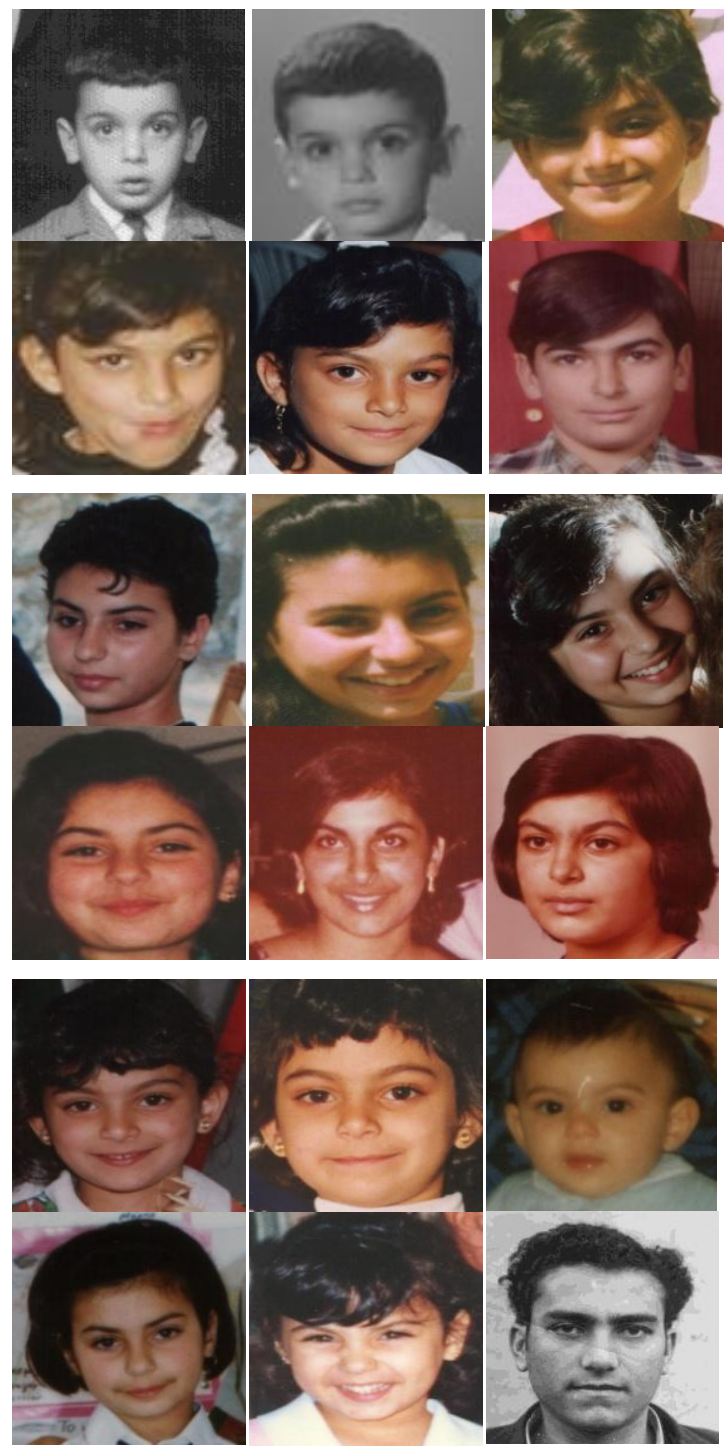

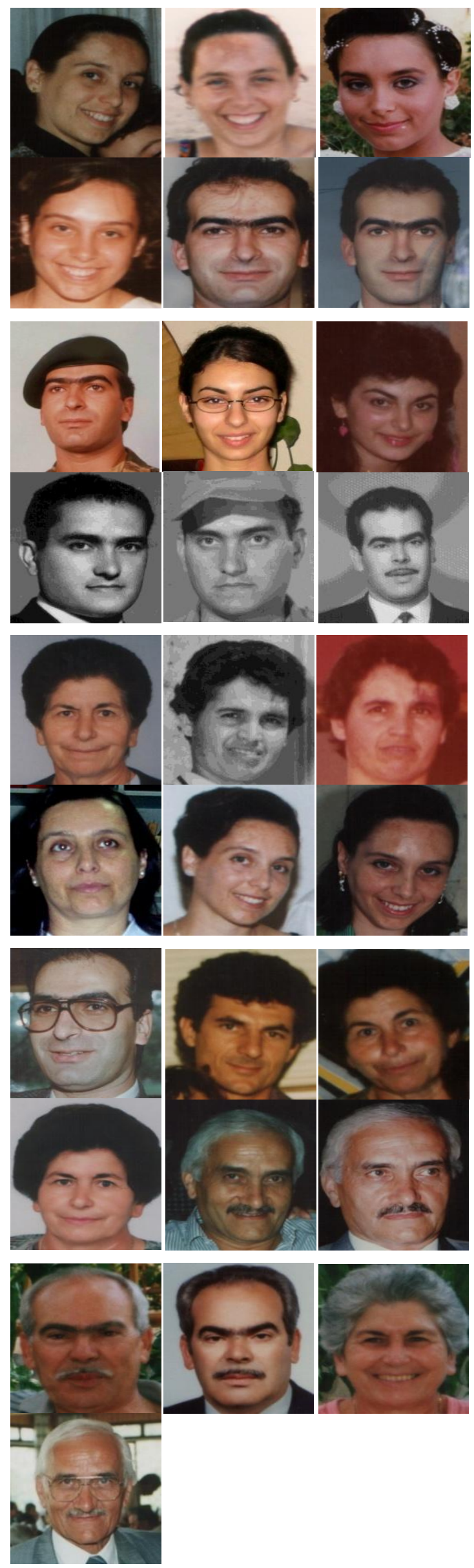

Figure 3: FGNET aging database: 011A07, 011A05, 010A10, 010A09, 010A07b, 001A14, 019A07, 009A14, 009A13, 009A11, 008A16, 008A13, 010A05, 010A04, 010A01, 009A09, 009A05, 004A21, 002A29, 002A26, 002A23, 002A21, 001A29, 001A28, 001A22, 009A22a, 008A21, 004A28, 004A26, 006A36, 005A40, 011A40, 001A43b, 002A31, 001A33 007A37, 005A52, 005A49, 004A53, 004A51, 048A54, 006А61, 005A61, 004A63.

Table 1: Feature set values of Child Age images.

\begin{tabular}{|c|c|c|c|c|c|}
\hline $\begin{array}{c}\text { S. } \\
\text { No }\end{array}$ & $\begin{array}{c}\text { Image } \\
\text { Name }\end{array}$ & Contrast & $\begin{array}{c}\text { Correla- } \\
\text { tion }\end{array}$ & Energy & $\begin{array}{c}\text { Homog- } \\
\text { eneity }\end{array}$ \\
\hline 1 & $001 \mathrm{~A} 02$ & 17.551 & 0.838 & 0.034 & 0.62953 \\
\hline 2 & $001 \mathrm{~A} 05$ & 15.478 & 0.859 & 0.042 & 0.66238 \\
\hline 3 & $001 \mathrm{~A} 08$ & 20.72 & 0.81 & 0.021 & 0.57649 \\
\hline 4 & $001 \mathrm{~A} 10$ & 24.445 & 0.613 & 0.023 & 0.53384 \\
\hline 5 & $002 \mathrm{~A} 03$ & 20.61 & 0.742 & 0.032 & 0.59142 \\
\hline 6 & $002 \mathrm{~A} 04$ & 23.384 & 0.652 & 0.03 & 0.56721 \\
\hline 7 & $002 \mathrm{~A} 07$ & 30.176 & 0.511 & 0.01 & 0.42063 \\
\hline 8 & $008 \mathrm{~A} 06$ & 21.905 & 0.752 & 0.02 & 0.5537 \\
\hline 9 & $009 \mathrm{~A} 00$ & 19.417 & 0.789 & 0.032 & 0.60454 \\
\hline 10 & $010 \mathrm{~A} 01$ & 19.347 & 0.746 & 0.029 & 0.57398 \\
\hline 11 & $010 \mathrm{~A} 09$ & 22.9 & 0.709 & 0.023 & 0.55456 \\
\hline 12 & $024 \mathrm{~A} 05$ & 27.495 & 0.539 & 0.013 & 0.45444 \\
\hline 13 & $024 \mathrm{~A} 10$ & 22.704 & 0.695 & 0.019 & 0.52015 \\
\hline 14 & $025 \mathrm{~A} 00$ & 12.626 & 0.967 & 0.05 & 0.70013 \\
\hline 15 & $025 \mathrm{~A} 03$ & 25.133 & 0.59 & 0.018 & 0.49319 \\
\hline 16 & $025 \mathrm{~A} 07$ & 26.681 & 0.558 & 0.016 & 0.48478 \\
\hline 17 & $002 \mathrm{~A} 12$ & 19.234 & 0.756 & 0.036 & 0.60803 \\
\hline 18 & $009 \mathrm{~A} 11$ & 16.487 & 0.9 & 0.03 & 0.64593 \\
\hline 19 & $025 \mathrm{~A} 12$ & 21.091 & 0.706 & 0.029 & 0.56771 \\
\hline 20 & $026 \mathrm{~A} 11$ & 23.454 & 0.669 & 0.021 & 0.53242 \\
\hline
\end{tabular}


Table 2 :Feature set values of Young Age images.

\begin{tabular}{|c|c|c|c|c|c|}
\hline $\begin{array}{l}\text { S. } \\
\text { No }\end{array}$ & $\begin{array}{l}\text { Image } \\
\text { Name }\end{array}$ & Contrast & $\begin{array}{c}\text { Correla } \\
\text {-tion }\end{array}$ & Energy & $\begin{array}{c}\text { Homog- } \\
\text { eneity }\end{array}$ \\
\hline 1 & 001A22 & 22.847 & 0.456 & 0.02 & 0.263 \\
\hline 2 & 001A28 & 29.051 & 0.526 & 0.026 & 0.314 \\
\hline 3 & 001A29 & 22.198 & 0.414 & 0.023 & 0.257 \\
\hline 4 & 003A23 & 28.624 & 0.497 & 0.031 & 0.311 \\
\hline 5 & 003A25 & 21.822 & 0.451 & 0.021 & 0.261 \\
\hline 6 & 012A21 & 25.679 & 0.285 & 0.014 & 0.169 \\
\hline 7 & 012A23 & 21.046 & 0.444 & 0.027 & 0.282 \\
\hline 8 & 012A24 & 22.977 & 0.352 & 0.02 & 0.222 \\
\hline 9 & 012A26 & 20.086 & 0.481 & 0.026 & 0.294 \\
\hline 10 & 012A27 & 28.563 & 0.505 & 0.033 & 0.218 \\
\hline 11 & 012A30 & 23.719 & 0.354 & 0.018 & 0.205 \\
\hline 12 & 024A23 & 22.342 & 0.413 & 0.023 & 0.247 \\
\hline 13 & 024A25 & 25.202 & 0.343 & 0.018 & 0.205 \\
\hline 14 & 027A22 & 25.297 & 0.296 & 0.016 & 0.181 \\
\hline 15 & 027A25 & 27.468 & 0.267 & 0.01 & 0.13 \\
\hline 16 & 027A30 & 22.272 & 0.403 & 0.023 & 0.246 \\
\hline 17 & $047 \mathrm{~A} 23$ & 23.665 & 0.315 & 0.03 & 0.264 \\
\hline 18 & 047A27 & 29.253 & 0.455 & 0.029 & 0.287 \\
\hline 19 & 048A30 & 27.441 & 0.269 & 0.014 & 0.165 \\
\hline 20 & 001A14 & 27.34 & 0.57 & 0.03 & 0.332 \\
\hline 21 & 001A16 & 32.474 & 0.182 & 0.012 & 0.128 \\
\hline 22 & 001A18 & 22.139 & 0.417 & 0.025 & 0.27 \\
\hline 23 & 001A19 & 28.197 & 0.235 & 0.015 & 0.174 \\
\hline 24 & 002A15 & 23.684 & 0.349 & 0.023 & 0.24 \\
\hline 25 & 009A13 & 27.407 & 0.269 & 0.013 & 0.153 \\
\hline 26 & 011A17 & 21.587 & 0.436 & 0.024 & 0.261 \\
\hline 27 & 011A20 & 29.685 & 0.469 & 0.029 & 0.298 \\
\hline 28 & 025A15 & 26.285 & 0.307 & 0.015 & 0.17 \\
\hline 29 & 025A18 & 24.958 & 0.316 & 0.017 & 0.195 \\
\hline 30 & 025A19 & 23.555 & 0.386 & 0.021 & 0.233 \\
\hline
\end{tabular}

Table 3: Feature set values of Middle Age images.

\begin{tabular}{|c|c|c|c|c|c|}
\hline $\begin{array}{c}\text { S. } \\
\text { No }\end{array}$ & $\begin{array}{l}\text { Image } \\
\text { Name }\end{array}$ & Contrast & $\begin{array}{c}\text { Correla } \\
\text {-tion }\end{array}$ & Energy & $\begin{array}{c}\text { Homog- } \\
\text { eneity }\end{array}$ \\
\hline 1 & 001A33 & 19.805 & 0.367 & 0.019 & 0.53 \\
\hline 2 & $001 \mathrm{~A} 40$ & 18.09 & 0.469 & 0.019 & 0.582 \\
\hline 3 & $002 \mathrm{~A} 31$ & 14.002 & 0.37 & 0.018 & 0.513 \\
\hline 4 & 003A35 & 16.716 & 0.592 & 0.02 & 0.638 \\
\hline 5 & 003A38 & 17.139 & 0.547 & 0.014 & 0.631 \\
\hline 6 & 012A32 & 13.988 & 0.345 & 0.017 & 0.495 \\
\hline 7 & 013A34 & 15.485 & 0.318 & 0.016 & 0.494 \\
\hline 8 & 018A33 & 18.772 & 0.471 & 0.018 & 0.616 \\
\hline 9 & 018A34 & 17.837 & 0.492 & 0.021 & 0.593 \\
\hline 10 & 019A37 & 18.586 & 0.393 & 0.015 & 0.549 \\
\hline 11 & 020A36 & 17.882 & 0.482 & 0.014 & 0.573 \\
\hline 12 & 021A35 & 17.004 & 0.288 & 0.012 & 0.447 \\
\hline 13 & 021A39 & 16.601 & 0.277 & 0.011 & 0.448 \\
\hline 14 & 025A34 & 17.18 & 0.243 & 0.012 & 0.445 \\
\hline 15 & 025A39 & 19.233 & 0.151 & 0.008 & 0.387 \\
\hline 16 & 039A35 & 16.244 & 0.268 & 0.015 & 0.47 \\
\hline 17 & 047A33 & 15.747 & 0.405 & 0.021 & 0.575 \\
\hline 18 & 001A43a & 15.886 & 0.316 & 0.018 & 0.531 \\
\hline 19 & 003A47 & 19.327 & 0.498 & 0.019 & 0.597 \\
\hline 20 & 003A49 & 19.582 & 0.485 & 0.019 & 0.601 \\
\hline 21 & 004A48 & 18.211 & 0.495 & 0.018 & 0.591 \\
\hline 22 & $005 \mathrm{~A} 45$ & 10.867 & 0.459 & 0.018 & 0.578 \\
\hline 23 & 006A42 & 13.81 & 0.365 & 0.02 & 0.541 \\
\hline 24 & 013A41 & 12.486 & 0.4 & 0.018 & 0.549 \\
\hline 25 & 013A44 & 13.879 & 0.372 & 0.019 & 0.524 \\
\hline 26 & 022A50 & 13.698 & 0.346 & 0.019 & 0.507 \\
\hline 27 & 028A46 & 15.795 & 0.316 & 0.02 & 0.54 \\
\hline 28 & 039A45 & 19.97 & 0.442 & 0.018 & 0.569 \\
\hline 29 & 039A50 & 14.547 & 0.336 & 0.011 & 0.516 \\
\hline 30 & 047A45 & 18.947 & 0.247 & 0.011 & 0.428 \\
\hline
\end{tabular}


Table 4: Feature set values of Senior Age images.

\begin{tabular}{|c|c|c|c|c|c|}
\hline $\begin{array}{c}\text { S. } \\
\text { No }\end{array}$ & $\begin{array}{l}\text { Image } \\
\text { Name }\end{array}$ & $\begin{array}{c}\text { Contras } \\
\mathbf{t}\end{array}$ & $\begin{array}{l}\text { Corre- } \\
\text { lation }\end{array}$ & Energy & $\begin{array}{c}\text { Homog } \\
\text { eneity }\end{array}$ \\
\hline 1 & 003A51 & 22.901 & 0.4314 & 0.0207 & 0.5557 \\
\hline 2 & 003A57 & 21.446 & 0.4721 & 0.0359 & 0.6277 \\
\hline 3 & 003A58 & 21.446 & 0.4317 & 0.0393 & 0.6212 \\
\hline 4 & 003A59 & 20.446 & 0.4993 & 0.037 & 0.6807 \\
\hline 5 & 003A60 & 24.376 & 0.3824 & 0.0186 & 0.5219 \\
\hline 6 & 004A53 & 20.855 & 0.4928 & 0.0277 & 0.5978 \\
\hline 7 & 006A54 & 23.698 & 0.4002 & 0.0194 & 0.5317 \\
\hline 8 & 006A55 & 23.698 & 0.4002 & 0.0194 & 0.5317 \\
\hline 9 & 006A56 & 23.698 & 0.4002 & 0.0194 & 0.5317 \\
\hline 10 & 039A52 & 24.426 & 0.3321 & 0.0182 & 0.5005 \\
\hline 11 & 047A55 & 23.694 & 0.3840 & 0.0214 & 0.5346 \\
\hline 12 & 004A62 & 22.667 & 0.4134 & 0.0213 & 0.5486 \\
\hline 13 & 004A63 & 20.642 & 0.4964 & 0.0265 & 0.607 \\
\hline 14 & 006A61 & 22.381 & 0.3727 & 0.0263 & 0.5651 \\
\hline 15 & 006A67 & 21.31 & 0.4640 & 0.0331 & 0.6038 \\
\hline 16 & 006A69 & 24.803 & 0.3712 & 0.018 & 0.5221 \\
\hline 17 & 045A64 & 21.326 & 0.4285 & 0.0266 & 0.5596 \\
\hline 18 & $045 \mathrm{~A} 65$ & 20.667 & 0.4000 & 0.0327 & 0.5879 \\
\hline 19 & 045A66 & 20.297 & 0.4421 & 0.0345 & 0.6012 \\
\hline 20 & 069A52 & 22.426 & 0.3321 & 0.0172 & 0.5005 \\
\hline
\end{tabular}

From the above tables derive an algorithm to classify the facial image into one of the category of Child age(0-12), Young Age(13-30), Middle Age(31-50) and Senior Age (51 70).

\section{Algorithm 1: Age group classification based on features} derived from CP-GLCM of TN-LBP

START

if Contrast > 20 and Homogeneity > 0.5

print( "Facial image is Senior Age (51-70)”)

else if Contrast $<20$ and energy $<0.022$

print(" Facial image is Middle Age (31-50)")

else if Contrast $>20$ and Homogeneity $<0.5$

print(" Facial image is Young Age (13-30)”)

else if Correlation > 0.5

print(" Facial image is child Age (0-12)")

else

print ("Facial image is unknown age group")

\section{COMPARISON OF THE PROPOSED METHOD WITH OTHER EXISTING METHODS}

The proposed method of age classification is compared with the existing methods [1,2]. The method proposed by $\mathrm{M}$. Yazdi et.al [1] classified age group using RBF Neural Network Classifier. The age group classification method proposed by Wen-Bing Horng is based on two geometric features and three wrinkle features of facial image. The percentage of classification of proposed method and other existing methods are listed in table 6. The graphical representation of the percentage mean classification rate for the proposed method and other existing methods are shown in Fig. 4.

Table 6: Classification rate of the proposed method with other existing methods.

\begin{tabular}{|l|c|c|c|}
\hline $\begin{array}{c}\text { Image } \\
\text { Dataset }\end{array}$ & $\begin{array}{c}\text { Existing age } \\
\text { classification } \\
\text { method [1] }\end{array}$ & $\begin{array}{c}\text { Existing age } \\
\text { classification } \\
\text { method [2] }\end{array}$ & $\begin{array}{c}\text { Proposed } \\
\text { Method }\end{array}$ \\
\hline FG-NET & 89.67 & 90.52 & 100 \\
\hline Google & 85.3 & 81.58 & 95 \\
\hline Scanned & 88.72 & 85.42 & 93.5 \\
\hline Average & 87.9 & 85.84 & 96.17 \\
\hline
\end{tabular}

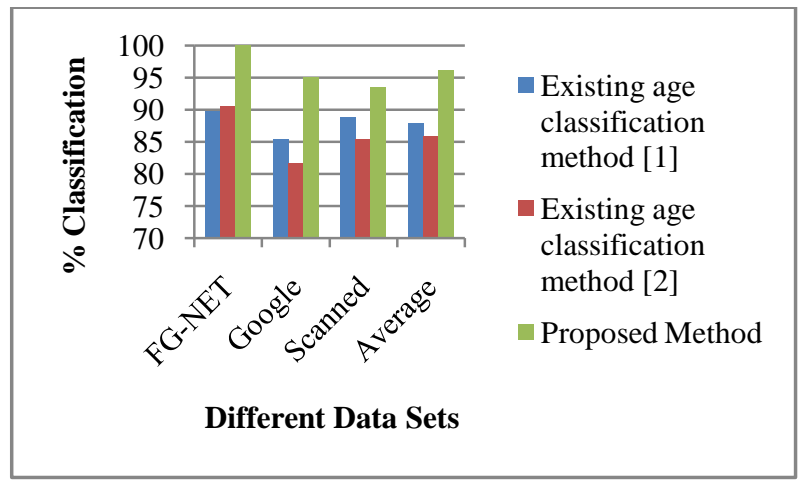

Figure 4: Classification chart of proposed method and other existing methods.

\section{CONCLUSIONS}

The proposed method estimated the relationship between the Top (outer) and Bottom (inner) corner pixels of TN-LBP. The bottom or inner corner pixels are connected and the top or outer corner pixels of TN-LBP are not connected. The proposed method present in this paper successively utilized the TN-LBP by reducing the complexity in establishing the LBP code for 13 pixels i.e. ranges from 0 to $2^{13}-1$. The proposed CP-GLCM of TN-LBP achieved a good classification rate when compared to the existing method. 
Table 5. Test set results of different dataset images.

\begin{tabular}{|c|c|c|c|c|c|c|c|}
\hline Sno & Image Name & Contrast & Correlation & Energy & Homogeneity & Group & Result \\
\hline 1 & gogle_im_01 & 25.202 & 0.5426 & 0.018 & 0.2049 & Middle & Success \\
\hline 2 & gogle_im_02 & 21.704 & 0.5945 & 0.019 & 0.5101 & Child & Success \\
\hline 3 & gogle_im_03 & 26.086 & 0.4806 & 0.026 & 0.2943 & Young & Success \\
\hline 4 & gogle_im_04 & 22.384 & 0.7519 & 0.03 & 0.5872 & Child & Success \\
\hline 5 & gogle_im_05 & 10.867 & 0.4975 & 0.019 & 0.5969 & Middle & Success \\
\hline 6 & gogle_im_06 & 23.694 & 0.3713 & 0.018 & 0.5221 & Senior & Success \\
\hline 7 & gogle_im_07 & 19.582 & 0.4853 & 0.019 & 0.6013 & Middle & Success \\
\hline 8 & gogle_im_08 & 21.326 & 0.4641 & 0.033 & 0.6038 & Senior & Success \\
\hline 9 & gogle_im_09 & 24.563 & 0.5054 & 0.033 & 0.4179 & Young & Success \\
\hline 10 & gogle_im_10 & 25.719 & 0.3542 & 0.018 & 0.4053 & Young & Success \\
\hline 11 & 076A14 & 26.342 & 0.4131 & 0.023 & 0.3466 & Young & Success \\
\hline 12 & 077A00 & 19.487 & 0.79 & 0.03 & 0.6459 & Child & Success \\
\hline 13 & 082A20 & 24.803 & 0.3727 & 0.026 & 0.5651 & Young & Success \\
\hline 14 & $082 \mathrm{~A} 25$ & 22.086 & 0.4806 & 0.026 & 0.2943 & Young & Success \\
\hline 15 & 067A33 & 15.886 & 0.3163 & 0.018 & 0.5306 & Middle & Success \\
\hline 16 & 067A39 & 13.81 & 0.4593 & 0.018 & 0.5782 & Middle & Success \\
\hline 17 & 048A52 & 19.327 & 0.3648 & 0.02 & 0.5413 & Middle & Success \\
\hline 18 & 048A54 & 21.31 & 0.4965 & 0.027 & 0.607 & Senior & Success \\
\hline 19 & 067A48 & 29.233 & 0.4046 & 0.021 & 0.5752 & Middle & Fail \\
\hline 20 & 048A65 & 20.905 & 0.7519 & 0.02 & 0.5537 & Child & Success \\
\hline 21 & sca.img-001 & 20.681 & 0.5576 & 0.016 & 0.4848 & Child & Success \\
\hline 22 & sca.img-002 & 20.046 & 0.4442 & 0.027 & 0.3224 & Young & Success \\
\hline 23 & sca.img-003 & 22.667 & 0.4286 & 0.027 & 0.5596 & Senior & Success \\
\hline 24 & sca.img-004 & 22.977 & 0.352 & 0.02 & 0.3124 & Young & Success \\
\hline 25 & sca.img-005 & 20.642 & 0.3841 & 0.021 & 0.5346 & Senior & Success \\
\hline 26 & sca.img-006 & 22.381 & 0.4134 & 0.021 & 0.5486 & Senior & Success \\
\hline 27 & sca.img-007 & 16.244 & 0.1508 & 0.008 & 0.3875 & Middle & Success \\
\hline 28 & sca.img-008 & 15.747 & 0.2682 & 0.015 & 0.4695 & Middle & Success \\
\hline 29 & sca.img-009 & 18.679 & 0.285 & 0.014 & 0.469 & Young & Fail \\
\hline 30 & sca.img-010 & 12.626 & 0.8667 & 0.05 & 0.7001 & Child & Success \\
\hline
\end{tabular}




\section{REFERENCES}

[1] M. Yazdi, S. Mardani-Samani, M. Bordbar, and R. Mobaraki "Age Classification based on RBF Neural Network" , Canadian Journal on Image Processing and Computer Vision Vol. 3 No. 2, pages: 38-42, June 2012.

[2] Wen-Bing Horng, Cheng-Ping Lee and Chun-Wen Chen "Classification of Age Groups Based on Facial Features", Tamkang Journal of Science and Engineering, Vol. 4, No. 3, pp. 183-192 Year: 2001.

[3] Ahonen T., Hadid A. and Pietikainen M., "Face Recognition with Local Binary Patterns," Computer Vision, ECCV Proceedings, pp. 469-481, 2004.

[4] Ahonen T., Hadid A., Pietikainen M., "Face description with local binary patterns: application to face recognition", IEEE Transactions on Pattern Analysis and Machine Intelligence, vol.28(12), pp.2037-2041, 2006.

[5] Chandra Mohan, VijayaKumar V., Damodaram A., "Adulthood classification based on geometrical facial features", ICGST-GVIP Journal, Volume 10, Issue 2, pp.39-44, June 2010 .

[6] Atkinson, P. M. and Lewis, P. "Geostatistical classification for remote sensing: An introduction", Comput. Geosci., Vol. 26, pp. 361-371, 2000

[7] Young H. Kwon and Niels da Vitoria Loboy, "Age Classification from Facial Images", Computer Vision and Image Understanding, Vol. 74, No. 1, pp. 1-21, April 1999.

[8] Sirovich L. and Kirby M. "Low-dimensional procedure for the characterization of human face," J. Opt. Am. A, vol.7, no.3, pp. 519-524, 1987.

[9] Hasegawa H. and Simizu E. "Discrimination of facial age generation using neural networks," T.IEE Japan, vol.117-C, no.12, pp.1897-1898, 1997.

[10] Kosugi M. "Human-face recognition using mosaic pattern and neural networks," IEICE Trans., vol.J76-DII, no.6, pp.1132-1139, June 1993.

[11] Jangala. Sasi Kiran, V. Vijaya Kumar and B. Eswara Reddy. "Age classifications based on second order image compressed and fuzzy reduced grey level (SICFRG) model," International Journal on Computer Science and Engineering (IJCSE), Vol. 5 No. 06, pp.481-492, Jun 2013.

[12] J Sasi Kiran, Dr. V. Vijaya Kumar and V V Harichandana, "An Effective Age Classification Using Topological Features Based on Compressed and Reduced Grey Level Model of the Facial Skin," I.J. Image,
Graphics and Signal Processing, Vol. 6, No. 1, pp. 9-17, November 2013.

[13] Dr. V. Vijaya Kumar, J. Sasi Kiran and Gorti Satyanarayana Murty, "Pattern based Dimensionality Reduction Model for Age Classification," International Journal of Computer Applications (IJCA), FCS Publishers, USA, Volume 79, No 13, pp. 14-20, October 2013.

[14] Gorti Satyanarayana Murty, J Sasi Kiran and Dr. V. Vijaya Kumar, "Facial Expression Recognition based on Features Derived from the Distinct LBP and GLCM," International Journal of Image, Graphics and Signal Processing(IJIGSP), MECS Publisher, Hong Kong, Vol. 6, No. 2, pp. 68-77, January 2014.

[15] Gorti Satyanarayana Murty, Dr. V. Vijaya Kumar and A. Obulesu, "Age classification based on simple LBP transitions," International Journal on Computer Science and Engineering (IJCSE), Vol. 5 No. 10, pp. 885-893, October 2013.

\section{AUTHOR'S PROFILE}

Pullela S V V S R Kumar is working as Associate Professor of CSE at V.S.Lakshmi Engineering College for Women. He received MCA Degree from Andhra University in 1998 and M.Tech (IT) from Punjabi University, Patiala in 2003. He is having more than 14 years of experience and published 8 research papers in various International Journals and Conferences. His research interests include Data Mining, Pattern Recognition and Image Processing. He is currently pursuing his Ph.D. from Acharya Nagarjuna University, Andhra Pradesh.

Dr. J V R Murthy is working as a Professor of CSE \& Director, Institute Industry Interaction Placement \& Training at University College of Engineering, Jawaharlal Nehru Technological University Kakinada, Kakinada. He received his B.Tech(EEE) from JNTU College of Engineering Kakinada. He received his M.Tech (CSE) for IIT, Karaghpur. $\mathrm{He}$ has completed his Ph.D from JNTU, Hydearbad in the year 2004. He worked as a senior consultant for KEYSPAN ENERGY, New York and AXA-Corporation, New York. His research interests include Data Mining, Cloud Computing and Information Retrieval Systems. He has produced 4 Ph.D.'s and produced more than 30 research papers in various National \& International Journals \& Conferences. He is a life member of ISTE.

V V Sahiti Srinidhi is pursuing her B.Tech. III Year I Semester CSE at SRM University, Chennai. She has participated in various Student symposiums and Seminars. Her research areas include Image Processing, Digital Watermarking and Cloud Computing. 